\title{
LETTER TO THE EDITOR \\ Reply: Evidence against volume conduction to explain normal MEPs in muscles with low motor power in $\mathrm{SCl}$
}

Spinal Cord (2014) 52, 718; doi:10.1038/sc.2014.117; published online 22 July 2014

We recently reported a case study of chronic spinal cord injury (SCI), in which motor evoked potentials (MEPs) with normal amplitude and latency could be recorded from the extensor carpi radials (ECR) muscle, even though the motor power of this muscle was just $1 / 5 .^{1}$

Calancie and Alexeeva ${ }^{2}$ have hypothesized that volume conduction from the unaffected brachioradialis (BR) muscle explains this observation, and suggested that this could be tested by recording the surface electromyography (EMG) from both muscles during a resisted elbow flexion with the forearm in mid-pronation and wrist relaxed.

We tested this hypothesis in the SCI participant from the original report. We observed strong EMG activity from the BR, whereas there was no EMG signal detected from the ECR (Figure 1). The root-mean-square EMG for BR was $97 \mu \mathrm{V}$ compared to $4 \mu \mathrm{V}$ for ECR (background noise level).

These results indicate that volume conduction from the BR does not explain our original report. The case study adds to previous literature $^{3}$ that small, delayed MEPs can be recorded from weak muscles in people with SCI, by demonstrating that in some cases these MEPs can be remarkably normal.

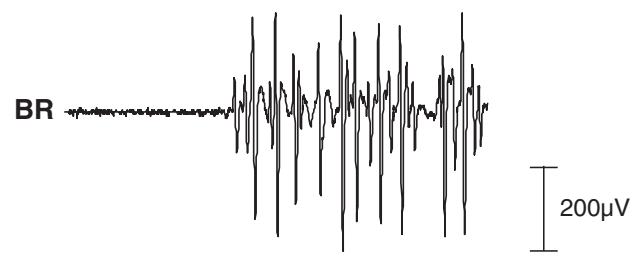

ECR

Figure 1 Surface EMG $(1 \mathrm{~s})$ from brachioradialis (BR) and extensor carpi radialis (ECR) during resisted elbow flexion. No volume conduction from $B R$ was detected in the ECR recording.

\section{CONFLICT OF INTEREST}

The authors declare no conflict of interest.

\section{ACKNOWLEDGEMENTS}

DJE, APL and MC were funded for this work by NIH grant R21HD077616.

DJ Edwards ${ }^{1,2,3}$, M Cortes ${ }^{1,4}$, GW Thickbroom ${ }^{2}$, A Rykman $^{1}$, A Pascual-Leone ${ }^{3,5}$ and BT Volpe ${ }^{6}$

${ }^{1}$ Non-invasive Brain Stimulation and Human Motor Control Laboratory, Departments of Neurology and Neuroscience, Burke Medical Research Institute, Weill Medical College of Cornell University, White

Plains, NY, USA;

${ }^{2}$ Australian Neuro-muscular Research Institute and Centre for Neuromuscular and Neurological Disorders, University of Western Australia, Perth, Western Australia, Australia

${ }^{3}$ Berenson-Allen Center for Non-Invasive Brain Stimulation, Harvard Medical School, Boston, MA, USA;

${ }^{4} E M G$ and Motor Control Unit, Department of Neurology, Hospital Clinic, Universitat de Barcelona, Barcelona, Spain

${ }^{5}$ Institut Guttmann, Universitat Autonoma de Barcelona, Barcelona, Spain and

${ }^{6}$ Feinstein Institute for Medical Research, Manhasset, NY, USA E-mail:dedwards@burke.org

1 Edwards DJ, Cortes M, Thickbroom GW, Rykman A, Pascual-Leone A, Volpe BT Preserved corticospinal conduction without voluntary movement after spinal cord injury. Spinal Cord 2013; 51: 765-767.

2 Calancie B, Alexeeva N. Response to 'Preserved corticospinal conduction without voluntary movement after spinal cord injury'. Spinal Cord 2014, 52: 717.

3 Sherwood AM, Dimitrijevic MR, McKay WB. Evidence of subclinical brain influence in clinically complete spinal cord injury: discomplete SCI. J Neurol Sci 1985; 110 90-98. 